\title{
Convergence Rate of Minimization Learning for Neural Networks
}

\author{
Marghny H. Mohamed, Teruya Minamoto, and Koichi Niijima \\ Kyushu University, Department of Informatics, Fukuoka, Kasuka 816, Japan, \\ Phone:+81-92-583-7635, Fax: +81-92-583-7635, \\ e-mail: mohamed@i.kyushu-u.ac.jp
}

\begin{abstract}
In this paper, we present the convergence rate of the error in a neural network which was learnt by a constructive method. The constructive mechanism is used to learn the neural network by adding hidden units to this neural network. The main idea of this work is to find the eigenvalues of the transformation matrix concerning the error before and after adding hidden units in the neural network. By using the eigenvalues, we show the relation between the convergence rate in neural networks without and with thresholds in the output layer.
\end{abstract}

\section{Introduction}

The size of a hidden layer in multilayer neural networks is one of the most important considerations when solving actual problems using the networks. There are many methods to reduce the structure of the networks such as destructive, constructive, and genetic algorithms (Weymaere and Martens, 1994).

Destructive or pruning methods start from a fairly large network and remove unimportant connections or units (Hassibi and Stork, 1993). Constructive or growth methods start from a small network and dynamically grow the network (Giles, Chen, Sun, Chen, Lee and Goudreau, 1995; Niijima et al., 1997). Constructive learning algorithms were used to handle multi-category classification with convergence to zero classification errors (Parekh et al., 1995). The advantage in using this method is that it can automatically find the size and the topology of the neural network without specifying them before training.

In the paper (Niijima et al., 1997), we proposed a learning algorithm which is carried out by mininmizing the erreor function to determine the topology of the neural network. This paper describes the convergence rate of our method. This analysis is carried out by finding the transformation matrix concerning the error before and after adding hidden units in the neural network. The key idea is to find the eigenvalues of this matrix. We consider two types of neural network without and with thresholds in the output layer. 


\section{Convergence Rate in a Neural Network without Thresholds in the Output Layer}

We consider a neural network which consists of an input layer with $n+1$ nodes, a hidden layer with $h$ units, and an output layer with $l$ units:

$$
y_{i}=g\left(\sum_{j=1}^{h} w_{i j} f\left(\sum_{k=1}^{n+1} v_{j k} x_{k}\right)\right), \quad i=1,2, \ldots, l,
$$

where $x_{k}$ indicates the $k$-th input value, $y_{i}$ the $i$-th output value, $v_{j k}$ a weight connecting the $k$-th input node with the $j$-th hidden unit, and $w_{i j}$ a weight between the $j$-th hidden unit and the $i$-th output unit. The functions $f(t)$ and $g(t)$ are given by $f(t)=(1-\exp (-t)) /(1+\exp (-t))$ and $g(t)=1 /(1+$ $\exp (-t))$, respectively. We write (1) as $y=g(W f(V x))$, where we set $x=$ ${ }^{t}\left(x_{1}, x_{2}, \ldots, x_{n}, x_{n+1}\right)$ with $x_{n+1}=-1, y={ }^{t}\left(y_{1}, y_{2}, \ldots, y_{l}\right), V=\left(v_{j k}\right)$ and $W=\left(w_{i j}\right)$. This network is shown in Fig.1 (a). Let $\left(x^{\nu}, y^{\nu}\right), \nu=1,2, \ldots, m$, be training data for the network. We define an output error between the outputs of the network for the imputs $x^{\nu}$ and the relevant outputs $y^{\nu}$ by

$$
J(V, W)=\sum_{\nu=1}^{m}\left\|g^{-1}\left(y^{\nu}\right)-W f\left(V x^{\nu}\right)\right\|^{2},
$$

where $g^{-1}\left(y^{\nu}\right)={ }^{t}\left(g^{-1}\left(y_{1}^{\nu}\right), g^{-1}\left(y_{2}^{\nu}\right), \ldots, g^{-1}\left(y_{l}^{\nu}\right)\right)$ with the inverse function $g^{-1}(s)$ of $s=g(t)$, and $\|\cdot\|$ stands for the Euclidean norm. To determine $V$ and $W$, we need to minimize the error function (2).

The paper (Niijima et al., 1997) presents a technique for determining the weights $V$ and $W$ successively by adding one unit to the hidden layer of this network. Let $\mathbf{v}$ denote a connection weight vector between the $(h+1)$-th hidden unit and the input layer, and let $w$ be a weight vector connecting the $(h+1)$-th hidden unit with the output layer. The neural network after adding the $(h+1)$ th hidden unit is shown in Fig.1 (b). We denote the weight matrices $(V, \mathbf{v})$ and $(W, \mathbf{w})$ by $\widetilde{V}$ and $\widetilde{W}$, respectively. Then a new error function can be written as

$$
J(\widetilde{V}, \widetilde{W})=\sum_{\nu=1}^{m}\left\|g^{-1}\left(y^{\nu}\right)-\widetilde{W} f\left(\widetilde{V} x^{\nu}\right)\right\|^{2}
$$

We describe how to determine the added weight vector $\mathbf{w}$. Since

$$
\widetilde{W} f\left(\tilde{V} x^{\nu}\right)=W f\left(V x^{\nu}\right)+\mathbf{w} f\left(\mathbf{v} x^{\nu}\right),
$$

the error function can be written as follows:

$$
J(\widetilde{V}, \widetilde{W})=J(V, W)-2\langle d, \mathbf{w}\rangle+a\|\mathbf{w}\|^{2}
$$

in which $d$ and $a$ denote $d=\sum_{\nu=1}^{m} f\left(\mathbf{v} x^{\nu}\right) c^{\nu}$ and $a=\sum_{\nu=1}^{m} f^{2}\left(\mathbf{v} x^{\nu}\right)$, where $c^{\nu}=$ $g^{-1}\left(y^{\nu}\right)-W f\left(V x^{\nu}\right)$ and the symbol $\langle\cdot, \cdot\rangle$ indicates an inner product in $R^{l}$. 


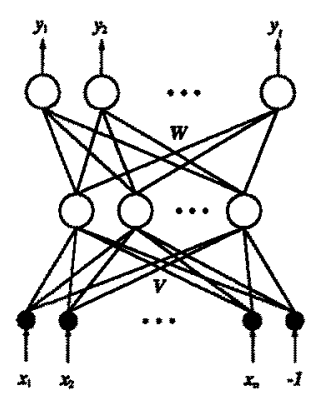

(a)

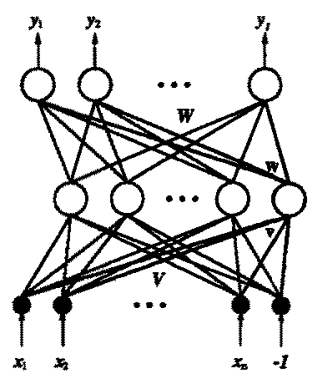

(b)

Fig. 1. (a) Neural network without thresholds in its output layer. (b) Neural network after adding one unit in the hidden layer in (a)

When the vector $\mathbf{v}$ is fixed, the vector $\mathbf{w}$ which minimizes the error function (3) is given by $w=d / a$. So the error after adding a hidden unit can be expressed as

$$
\widetilde{c}^{\nu}=c^{\nu}-\frac{d}{a} f\left(\mathbf{v} x^{\nu}\right)
$$

Furthermore, let ${ }^{t} \widetilde{C_{i}}=\left(c_{i}^{1}, c_{i}^{2}, \ldots, c_{i}^{m}\right),{ }^{t} C_{i}=\left(c_{i}^{1}, c_{i}^{2}, \ldots, c_{i}^{m}\right)$ and ${ }^{t} S=$ $\left(s_{1}, s_{2}, \ldots, s_{m}\right)$ with $s_{\nu}=f\left(v x^{\nu}\right)$ and so we can get

$$
{ }^{t} \widetilde{C_{i}}={ }^{t} C_{i}\left(E_{m}-\frac{1}{a} S^{t} S\right)={ }^{t} C_{i} \Gamma_{1}(\mathbf{v})
$$

where $\Gamma_{1}(\mathbf{v})=E_{m}-S^{t} S / a$ and $E_{m}$ is the unit matrix. The matrix $\Gamma_{1}(\mathbf{v})$ is symmetric and has various remarkable characterizations as in the following theorem.

Theorem 1. The matrix $\Gamma_{1}(\mathrm{v})$ satisfies $0 \leq\left\langle\Gamma_{1}(\mathrm{v}) U, U\right\rangle \leq\|U\|^{2}$ and the eigenvalues $\lambda_{j}$ of this matrix are given by

$$
\lambda_{1}=1, \lambda_{2}=1, \ldots, \lambda_{m-1}=1, \lambda_{m}=0 .
$$

The proof can be found in (Mohamed, et al., 1997). These eigenvalues mean that the convergence rate of errors before and after adding hidden units is not depending on the connection weights $v$ between the hidden layer and input layer.

\section{Convergence Rate in a Neural Network with Thresholds in the Output Layer}

We consider the network with thresholds $\theta_{i}, i=1,2 \ldots, l$, in its output layer as shown in Fig.2 (c). In this case, we can write

$$
y_{i}=g\left(\sum_{j=1}^{h} w_{i j} f\left(\sum_{k=1}^{n+1} v_{j k} x_{k}\right)-\theta_{i}\right), \quad i=1,2, \ldots, l
$$


whose simple form is $y=g(W f(V x)-\theta)$, where $\theta=\left(\theta_{1}, \theta_{2}, \ldots, \theta_{l}\right)$. The error function related to the present network takes the following form

$$
J(V, W, \theta)=\sum_{\nu=1}^{m}\left\|g^{-1}\left(y^{\nu}\right)-W f\left(V x^{\nu}\right)+\theta\right\|^{2} .
$$

We add one unit to the hidden layer and represent new weight vectors again by $\mathbf{v}$ and $\mathbf{w}$. By adding one hidden unit, the threshold vector $\theta$ must be changed. We denote a new threshold by $\widetilde{\theta}$ and write as $\widetilde{\theta}=\theta+\Delta \theta$. The network after adding one hidden unit is shown in Fig.2 (d). The error function related to this network can be expressed as

$$
J(\widetilde{V}, \widetilde{W}, \widetilde{\theta})=\sum_{\nu=1}^{m}\left\|g^{-1}\left(y^{\nu}\right)-\widetilde{W} f\left(\widetilde{V} x^{\nu}\right)+\widetilde{\theta}\right\|^{2},
$$

where we have used again the symbols $\widetilde{V}=(V, \mathbf{v})$ and $\widetilde{W}=(W, \mathbf{w})$.

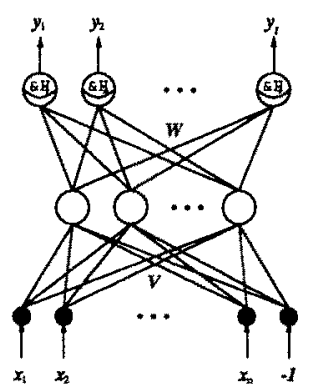

(c)

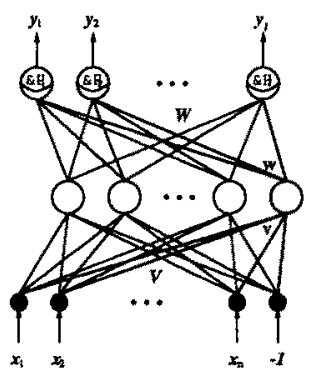

(d)

Fig. 2. (c) Neural network with thresholds in its output layer. (d) Neural network after adding one unit in the hidden layer in (c)

The same procedure as in the previous section will be applied in order to determine $\mathbf{w}$ and $\Delta \theta$ so that $J(\widetilde{V}, \widetilde{W}, \widetilde{\theta})$ is minimum. Since $\widetilde{W} f\left(\widetilde{V} x^{\nu}\right)=$ $W f\left(V x^{\nu}\right)+\mathbf{w} f\left(\mathbf{v} x^{\nu}\right)$ and $\tilde{\theta}=\theta+\Delta \theta$, we can decompose the error function $J(\widetilde{V}, \widetilde{W}, \widetilde{\theta})$ as

$$
\begin{aligned}
J(\widetilde{V}, \widetilde{W}, \widetilde{\theta})= & J(V, W, \theta)-2 \sum_{\nu=1}^{m} f\left(\mathbf{v} x^{\nu}\right)\left\langle q^{\nu}+\Delta \theta, \mathbf{w}\right\rangle \\
& +\sum_{\nu=1}^{m} f^{2}\left(\mathbf{v} x^{\nu}\right)\|\mathbf{w}\|^{2}+2 \sum_{\nu=1}^{m}\left\langle\Delta \theta, q^{\nu}\right\rangle+\sum_{\nu=1}^{m}\|\Delta \theta\|^{2},
\end{aligned}
$$

where

$$
q^{\nu}=g^{-1}\left(y^{\nu}\right)-W f\left(V x^{\nu}\right)+\theta .
$$

By minimizing this error function, we can easily determine $\mathbf{w}$ and $\Delta \theta$ as

$$
\mathbf{w}=\frac{m d_{1}-a_{2} d_{2}}{b}, \quad \Delta \theta=\frac{a_{2} d_{1}-a_{1} d_{2}}{b},
$$


where $a_{1}=\sum_{\nu=1}^{m} f^{2}\left(\mathbf{v} x^{\nu}\right), a_{2}=\sum_{\nu=1}^{m} f\left(\mathbf{v} x^{\nu}\right), d_{1}=\sum_{\nu=1}^{m} f\left(\mathbf{v} x^{\nu}\right) q^{\nu}, d_{2}=$ $\sum_{\nu=1}^{m} q^{\nu}$, and $b=m a_{1}-a_{2}^{2}$. Now we consider the convergence rate of the error as in the previous section. Since

$$
\widetilde{q}^{\nu}=q^{\nu}-\mathbf{w} f\left(\mathbf{v} x^{\nu}\right)+\Delta \theta
$$

we can write the error at the $i$-th component in the output layer as

$$
\widetilde{q}_{i}^{\nu}=q_{i}^{\nu}-\mathbf{w}_{i} f\left(\mathbf{v} x^{\nu}\right)+\triangle \theta_{i} .
$$

From (4), the $i$-th component of $\mathrm{w}$ and $\Delta \theta$ can be written as

$$
\mathbf{w}_{i}={ }^{t} Q_{i}\left[\frac{m}{b} S-\frac{a_{2}}{b} \mathbf{1}\right], \quad \Delta \theta_{i}={ }^{t} Q_{i}\left[\frac{a_{2}}{b} S-\frac{a_{1}}{b} \mathbf{1}\right],
$$

where ${ }^{t} \widetilde{Q}_{i}=\left(\tilde{q}_{i}^{1}, \widetilde{q}_{i}^{2}, \ldots, \widetilde{q}_{i}^{n}\right),{ }^{t} Q_{i}=\left(q_{i}^{1}, q_{i}^{2}, \ldots, q_{i}^{m}\right),{ }^{t} S=\left(s_{1}, s_{2}, \ldots, s_{m}\right)$, and ${ }^{t} \mathbf{1}=(1,1, \ldots, 1)$ with $s_{\nu}=f\left(\mathbf{v} x^{\nu}\right)$. Hence, we have by (5),

$$
{ }^{t} \widetilde{Q}_{i}={ }^{t} Q_{i}\left[E_{m}-\frac{m}{b} S{ }^{t} S+\frac{a_{2}}{b} 1{ }^{t} S+\frac{a_{2}}{b} S{ }^{t} \mathbf{1}-\frac{a_{1}}{b} 1^{t} \mathbf{1}\right]={ }^{t} Q_{i} \Gamma_{2}(\mathbf{v}) .
$$

Theorem 2. The matrix $\Gamma_{2}(\mathbf{v})$ satisfies $-\|U\|^{2} \leq\left\langle\Gamma_{2}(\mathbf{v}) U, U\right\rangle \leq\|U\|^{2}$ and the eigenvalues $\lambda_{j}$ of this matrix are given by

$$
\lambda_{1}=1, \lambda_{2}=1, \ldots, \lambda_{m-2}=1, \lambda_{m-1}=0, \lambda_{m}=0 .
$$

The proof can be found in (Mohamed, et al., 1997). This theorem show that the error after determining the weight $w$ and the correction $\Delta \theta$ between the hidden unit and output layer converges and its convergence rate is not depending on $\mathbf{v}$.

\section{Simulations}

Based on the proposed algorithm (Niijima, et al., 1997), we constructed a neural network without and with thresholds for 40 pictures, part of them are illustrated below. A summary of the number of hidden units generated for each pattern with $30 \%$ added noise appears in Tab. 1 and 2 for the network without and with thresholds, respectively. From these results we see that the network with thresholds performs better than without thresholds.
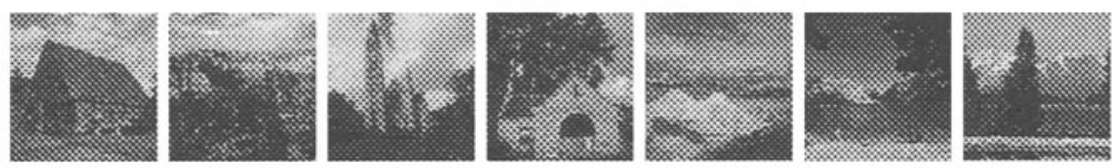

Fig. 3. Part of the memorized patterns 
Table 1. Simulation results for the neural network in Sect.2

\begin{tabular}{llllllllllllllllllll}
\hline 6 & 10 & 2 & 5 & 9 & 4 & 7 & 3 & 7 & 9 & 8 & 2 & 7 & 5 & 5 & 3 & 5 & 6 & 10 & 7 \\
$x^{1}$ & $x^{2}$ & $x^{3}$ & $x^{4}$ & $x^{5}$ & $x^{6}$ & $x^{7}$ & $x^{8}$ & $x^{9}$ & $x^{10}$ & $x^{11}$ & $x^{12}$ & $x^{13}$ & $x^{14}$ & $x^{15}$ & $x^{16}$ & $x^{17}$ & $x^{18}$ & $x^{19}$ & $x^{20}$ \\
\hline 6 & 8 & 7 & 5 & 4 & 7 & 5 & 6 & 10 & 5 & 5 & 7 & 4 & 10 & 3 & 5 & 8 & 8 & 5 & 4 \\
$x^{21}$ & $x^{22}$ & $x^{23}$ & $x^{24}$ & $x^{25}$ & $x^{26}$ & $x^{27}$ & $x^{28}$ & $x^{29}$ & $x^{30}$ & $x^{31}$ & $x^{32}$ & $x^{33}$ & $x^{34}$ & $x^{35}$ & $x^{36}$ & $x^{37}$ & $x^{38}$ & $x^{39}$ & $x^{40}$ \\
\hline
\end{tabular}

Table 2. Simulation results for the neural network in Sect.3

\begin{tabular}{llllllllllllllllllll}
\hline 6 & 10 & 4 & 7 & 6 & 3 & 6 & 3 & 8 & 8 & 5 & 4 & 5 & 5 & 1 & 4 & 5 & 4 & 8 & 3 \\
$x^{1}$ & $x^{2}$ & $x^{3}$ & $x^{4}$ & $x^{5}$ & $x^{6}$ & $x^{7}$ & $x^{8}$ & $x^{9}$ & $x^{10}$ & $x^{11}$ & $x^{12}$ & $x^{13}$ & $x^{14}$ & $x^{25}$ & $x^{16}$ & $x^{17}$ & $x^{18}$ & $x^{19}$ & $x^{20}$ \\
\hline 4 & 4 & 7 & 6 & 4 & 3 & 5 & 4 & 6 & 5 & 2 & 7 & 4 & 10 & 3 & 5 & 6 & 9 & 2 & 7 \\
$x^{21}$ & $x^{22}$ & $x^{23}$ & $x^{24}$ & $x^{25}$ & $x^{26}$ & $x^{27}$ & $x^{28}$ & $x^{29}$ & $x^{30}$ & $x^{31}$ & $x^{32}$ & $x^{33}$ & $x^{34}$ & $x^{35}$ & $x^{36}$ & $x^{37}$ & $x^{38}$ & $x^{39}$ & $x^{40}$ \\
\hline
\end{tabular}

\section{Conclusion}

In this paper, we presented a novel approach to extract the convergence rate for neural networks which were learnt by a constructive method. This approach is carried out by finding the eigenvalues of the transformation matrix concerning the error before and after adding hidden units. The results show that this error is convergent in both networks without and with thresholds in the output layer, and not depending on the connection weights between the hidden layer and input layer. Moreover, the performance of the network with thresholds is better than that without thresholds in its output layer.

\section{References}

[1] Giles, C.J., Chen, D., Sun, G., Chen, H., Lee, Y., and Goudreau, M.W.: Constructive learning of recurrent neural networks: Limitations of recurrent cascade correlation and a simple solution. IEEE Trans. Neural Networks. 6(1)(1995) 829-836

[2] Hassibi, B., Stork, D. G.: Second order derivatives for network pruning: Optimal brain surgeon. In S.J. Hanson, J.D.Cowan, C.L. Giles (Eds.). Advances in Neural Information Processing Systems. 5(1993) 164-171, San Mateo, CA: Morgan Kaufmann

[3] Mohamed, H.M., Minamoto, T., and Nijima, K.: Convergence rate of minimization learning for neural networks. DOI Technical Report, DOI-TR-141, Kyushu University, (1997)

[4] Niijima, K., Yamada, M., Mohamed,H.M., Akanuma, T., Minamoto, T., and Ohkubo, A.: Minimization learning of neural networks by adding hidden units. Research Reports on Information Science and Electrical Engineering of Kyushu University. 2(2)(1997) 173-178

[5] Parekh, R., Yang, J., Honavar, V.: Constructive Neural Networks Learning Algorithms for Multi-Category Pattern Classification. Technical Report TR95-15, AI Research Group, Dept. of Computer Science, Iowa State University

[6] Weymaere, N., and Martens, J.: On the initialization and optimization of multilayer perceptrons. IEEE Trans. Neural Networks. 5(5)(1994) 738-751 